\title{
Using Lifetime and Quenching Rate Constant to Determine Quencher Concentration
}

Xena Soto $^{a, b}$, John R. Swierk ${ }^{a *}$

a. Department of Chemistry, State University of New York at Binghamton, Vestal, NY 13850

b. Department of Chemistry, Lehman College / City University of New York, Bronx, NY 10468

\author{
AUTHOR INFORMATION \\ Corresponding Author \\ *jswierk@binghamton.edu
}


ABSTRACT Excited state quenching is a key step in photochemical reactions that involve energy or electron transfer. High reaction quantum yields require sufficiently high concentrations of quencher to ensure efficient quenching. Determination of quencher concentrations is typically done through trial and error. Using kinetic modeling, however, a simple relationship was developed that predicts the concentration of quencher necessary to quench $90 \%$ of excited states, using only the photosensitizer lifetime and the rate constant for quenching as inputs. Comparison of predicted quencher concentrations and quencher concentrations used in photoredox reactions featuring acridinium-based photocatalysts reveals that the majority of reactions used quencher concentrations significantly below the predicted concentration. This suggests these reactions exhibit low quantum yields, requiring long reaction times and/or intense light sources.

\section{TOC GRAPHICS}

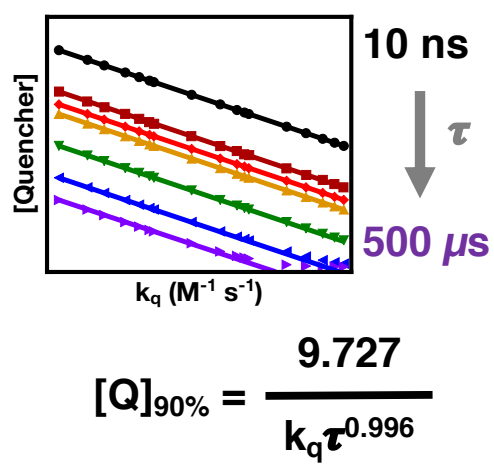

KEYWORDS Stern-Volmer, Kinetic Modeling, Photochemistry, Quantum Yield, Photoredox 
Quenching an excited state is a key step in photochemical reactions that involve electron or energy transfer. ${ }^{1}$ Poor kinetics at this step lead to inefficient harvesting of excited states and limit the overall quantum yield of the reaction. As a second order process, the rate of quenching depends on the concentration of excited photosensitizers, the concentration of the quencher (Q), and the rate constant for quenching $\left(\mathrm{k}_{\mathrm{q}}\right)$. The quenching step, however, is in direct competition with the unproductive relaxation of the excited state, which is controlled by the lifetime of the photosensitizer $(\tau)$. From a reaction design standpoint, the choice of photosensitizer controls $\tau$, though other factors such as redox potentials and absorption range often take higher priority. ${ }^{2}$ The value of $\mathrm{k}_{\mathrm{q}}$ depends on a host of factors including the choice of substrate, driving force for electron/energy transfer, and degree of association in solution..$^{3-5}$ While $\mathrm{k}_{\mathrm{q}}$ can also be tuned in a number of ways (e.g., changing photosensitizer or substrate, changing solvent, adding inert salt) these can be impractical from a reaction design standpoint where specific reaction conditions are needed to produce a given product, simplify purification, or solubilize one or more reagents. In principle the concentration of excited photosensitizers can be varied with light intensity, though as shown below that has little impact on the quenching yield. Thus, the most practical parameter that can be varied to impact quenching rates and efficiency is the concentration of quencher. However, a method to quantitatively predict the quencher concentration needed for efficient quenching does not currently exist. Instead, trial and error is typically used to determine optimal concentration.

Inspired by our recent success using kinetic modeling to reproduce reaction quantum yields, we hypothesized that with knowledge of $\mathrm{k}_{\mathrm{q}}$ and $\tau$ kinetic modeling could be used to develop a simple, predictive model for quencher concentration. ${ }^{6,7}$ Photosensitizer lifetimes are widely reported for both organic and inorganic photosensitizers. ${ }^{1,5}$ Determination of $\mathrm{k}_{\mathrm{q}}$ for a 
photochemical reaction is simple using the Stern-Volmer relationship and commonly reported. In the simplest formulation of Stern-Volmer, all that is needed is a fluorimeter, emissive photosensitizer, and knowledge of $\tau$ to determine a value for $\mathrm{k}_{\mathrm{q}}$.

Using Kinetiscope, ${ }^{8}$ a freeware stochastic kinetics simulator widely used to study chemical reactions, ${ }^{9-11}$ we developed a simple model that involved competition between relaxation of the excited state and quenching by a quencher, Q. Our specific reaction involved an oxidative quenching reaction that generated a reduced quencher, $\mathrm{Q}^{\circ-}$, and oxidized photosensitizer, however, the result would be unchanged for a reductive quenching or energy transfer. Relaxation, $\mathrm{k}_{\text {relax }}$, is a first order process controlled by $\tau\left(\mathrm{k}_{\text {relax }}=1 / \tau\right)$, while $\mathrm{k}_{\mathrm{q}}$ was varied from $10^{7} \mathrm{M}^{-1} \mathrm{~s}^{-1}$ to $10^{10} \mathrm{M}^{-1} \mathrm{~s}^{-1}$. Values of $\mathrm{k}_{\mathrm{q}}$ greater than $10^{10} \mathrm{M}^{-1} \mathrm{~s}^{-1}$ were not explored as that indicates a reaction that is not diffusion controlled and requires pre-association of photosensitizer and quencher or an intramolecular energy/electron transfer. Initially, we modeled continuous illumination and varied the concentration of $Q$ to achieve a quantum yield for quenching, $\Phi_{\text {quench }}$, of $0.900 \pm 0.001$ at the end of the simulation. In this case, $\Phi_{\text {quench }}$ is defined as the final concentration of $\left[\mathrm{Q}^{-}\right]$divided by the number of photons introduced to the reaction. Targeting a value of $0.900 \pm 0.001$ for $\Phi_{\text {quench }}$ represented an optimal balance of harvesting a high concentration of excited states at quencher concentrations that could be practical. We did not regenerate the oxidized photosensitizer in our reaction, however the concentration of photosensitizer was relatively high $(100 \mu \mathrm{M})$, length of the simulation was kept short $(1 \mathrm{~s})$, and the illumination intensity kept to $1.63 \times 10^{-5}$ photons/s, which corresponds to a $10 \mathrm{~mW}$ intensity of $415 \mathrm{~nm}$ light. As a result, only a relatively small concentration of photosensitizer was oxidized during the simulation. In addition, increasing both the simulation time and illumination intensity 
(Figure S1 and S2) resulted in no change in $\Phi_{\text {quench, }}$ indicating that the buildup of oxidized photosensitizer has negligible impact on the reaction.
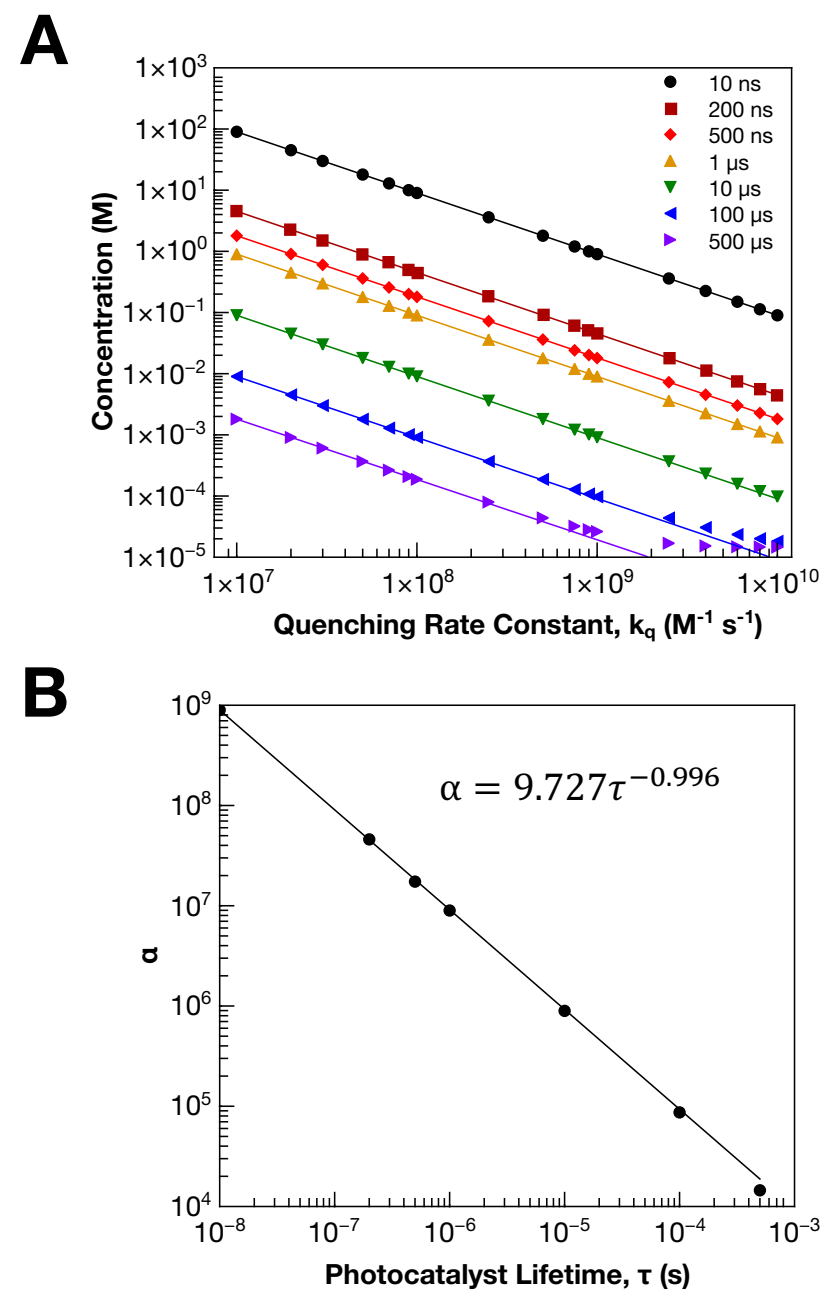

Figure 1. A) Predicted concentration of quencher needed to achieve quenching of $90 \%$ of excited states as a function of quenching rate constant $\left(\mathrm{k}_{\mathrm{q}}\right)$ and photosensitizer lifetime. Modeled with continuous illumination of $1.63 \times 10^{-5}$ photons/s. Solid lines are fit to power law equation of the general form $\mathrm{k}_{\mathrm{q}} / \alpha$. B) Value of $\alpha$ as a function of photosensitizer lifetime $(\tau)$. Solid black line is fit to equation: $\alpha=9.727 \tau^{-0.996}$. 
As shown in Figure 1A, varying $\mathrm{k}_{\mathrm{q}}$ for a given value of $\tau$ resulted in a 2-3 order of magnitude variation in the predicted concentration of quencher. To our delight, for a given photosensitizer lifetime, the predicted values of [Q] could be fit to a simple power law equation of the form:

$[\mathrm{Q}]=\alpha / \mathrm{k}_{\mathrm{q}}$

At short values of $\tau$, the fit to equation 1 was excellent with an $\mathrm{R}^{2}$ value of 1 . At longer values of $\tau$ (e.q., $500 \mu \mathrm{s}$ ) the fit was less accurate at larger $\mathrm{k}_{\mathrm{q}}$ values $\left(>10^{9} \mathrm{M}^{-1} \mathrm{~s}^{-1}\right)$. This is because at those values, the concentration of quencher needed was essentially independent of $\mathrm{k}_{\mathrm{q}}$ due to the extremely large values of $\tau$. Figure 1B shows a plot of the value of $\alpha$ as a function of $\tau$ and demonstrates that data could also be described by a power law equation of the form:

$\alpha=\mathrm{A} / \tau^{\mathrm{B}}$

From the fit in Figure 1B, values of 9.727 and 0.996 were determined for A and B, respectively. Thus, combining equations 1 and 2 , as well as the values of $\mathrm{A}$ and $\mathrm{B}$, the concentration of $\mathrm{Q}$ needed to give a $\Phi_{\text {quench }}$ of 0.9 at a given value of $\tau$ and $\mathrm{k}_{\mathrm{q}}$ is given by equation 3 :

$[Q]=\frac{9.727}{\mathrm{k}_{\mathrm{q}} \tau^{0.996}}$

For values of $\tau$ less than or equal to $10 \mu \mathrm{s}$, the deviation between [Q] predicted from kinetic modeling and $[\mathrm{Q}]$ predicted by equation 3 was $5 \%$ or less and typically $1-2 \%$ at most. For longer values of $\tau$, the values predicted by equation 3 showed significant deviation at values of $k_{q}$ less than $10^{9} \mathrm{M}^{-1} \mathrm{~s}^{-1}$ and much small deviations at smaller values of $\mathrm{k}_{\mathrm{q}}$. Percent deviations are shown in Table S1. We note that the majority of photosensitizers used in photochemical reactions have 
$\tau$ on the order of $10 \mu$ s or shorter and that in the cases of longer $\tau$ values, equation 3

overestimates the concentration of Q needed, meaning that a $\Phi_{\text {quench }}$ higher than 0.9 would be expected.

If we consider the classic Stern-Volmer model of the form:

$\frac{I_{0}}{I}=1+k_{q} \tau[Q]$

and assume our $\Phi_{\text {quench }}$ of 0.9 , then equation 4 rearranges into:

$[Q]=\frac{9}{k_{q} \tau}$

Equation 4 is in reasonably good agreement with equation 3 and deviates primarily because of the poor fit of the 100 and $500 \mu$ s data at large $\mathrm{k}_{\mathrm{q}}$ to the power law model. It is important to note that the classic, linear form of the Stern-Volmer equation merely allows for an estimation of $\mathrm{k}_{\mathrm{q}}$ but fails to consider a host of effects, including diffusion. ${ }^{12}$

We also performed a similar analysis as above but started with a fixed concentration of excited photosensitizer. This simulates pulsed illumination, like in a laser experiment, where a brief, intense pulse of light excites a significant number of photosensitizers followed by a dark period. As our benchmark, we set a value of $\Phi_{\text {quench }}$ of 0.9 after 100 ns of reaction time. Again, this represented a balance between a high value of $\Phi_{\text {quench }}$ and reasonable concentrations of Q. We also limited our investigation to photosensitizers with lifetimes of $200 \mathrm{~ns}$ to $10 \mu \mathrm{s}$. As with continuous illumination, the data could be well fit to equation 1 (Figure S3), however the plot of $\alpha$ versus $\tau$ did not follow the form of equation 2 (Figure S4). As $\tau$ gets longer Figure $\mathbf{S 3}$ shows 
that $\Phi_{\text {quench }}$ has less of a concentration dependence, likely because relaxation becomes a minor unproductive pathway on the timescale of $100 \mathrm{~ns}$.

In order to validate our modeling, we calculated $\Phi_{\text {quench }}$ for a group of experimental systems described in the literature and compared the predicted $\Phi_{\text {quench }}$ with the measured $\Phi_{\text {quench }}$. Measurement of $\Phi_{\text {quench }}$ by itself is not common and accurate measurements can be challenging because of rapid back electron transfer, low cage escape yields, or other unproductive pathways. ${ }^{13-15}$ Mindful of this, we carefully selected a set of trial reactions where $\Phi_{\text {quench }}$ was known independently of other unproductive pathways. Figure 2 shows the comparison between predicted and measured $\Phi_{\text {quench }}$ and demonstrates an excellent correlation, suggesting that our kinetic modeling method produces values in good agreement with experiment.

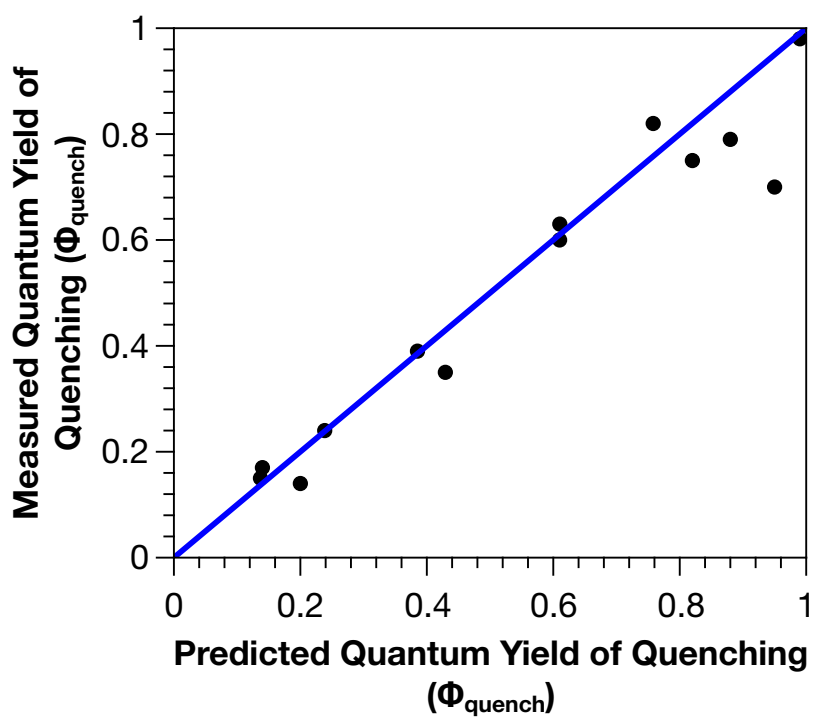

Figure 2. Predicted quantum yield of quenching $\left(\Phi_{\text {quench }}\right)$ compared to experimentally measured quantum yields of quenching $\left(\Phi_{\text {quench }}\right)$. Solid blue line shows one to one correlation. Details for measured quantum yields are in the supporting information. 
Equation 3 can also be a useful tool in evaluating reaction design. Using a series of photoredox reactions that rely on acridinium-based photocatalysts, we compared the quencher concentrations used in experimental reports to the concentration of quencher predicted by equation 3. Acridinium-based photocatalysts are commonly used in photoredox reactions and typically exhibit short lifetimes on the order of $10 \mathrm{~ns} .{ }^{16}$ Figure 3 shows that the majority of experimental reports we evaluated used less quencher than equation 3 predicts is needed for efficient quenching. It is also notable that deviation becomes more pronounced at smaller values of $\mathrm{k}_{\mathrm{q}}$. This is largely a function of most of reports using a quencher concentration on the order of 0.1 to $0.2 \mathrm{M}$. While this concentration range is appropriate for a larger values of $\mathrm{k}_{\mathrm{q}}$ of $\left(10^{9} \mathrm{M}^{-1} \mathrm{~s}\right.$ ${ }^{1}$ or greater), it is too low for smaller values of $\mathrm{k}_{\mathrm{q}}$. It is important to note that using a quencher concentration that is too low will be impact the quantum yield of the reaction but not necessarily the overall product yield and in fact, most of the reactions surveyed in Figure 3 achieve product yields of $70 \%$ or higher. However, the majority of the reactions ran for more than 24 hours and utilized extremely bright light sources with illumination intensities on the order of watts. This suggests that the quantum yields of these reactions are indeed low and may prove to be an issue when considering the energy intensity of these photoredox reactions.${ }^{17}$ More generally, this suggests that photoredox reactions that rely on photocatalysts with short lifetimes on the nanosecond timescale will struggle to achieve high quantum yields unless paired with substrates that exhibit a large $\mathrm{k}_{\mathrm{q}}$. 


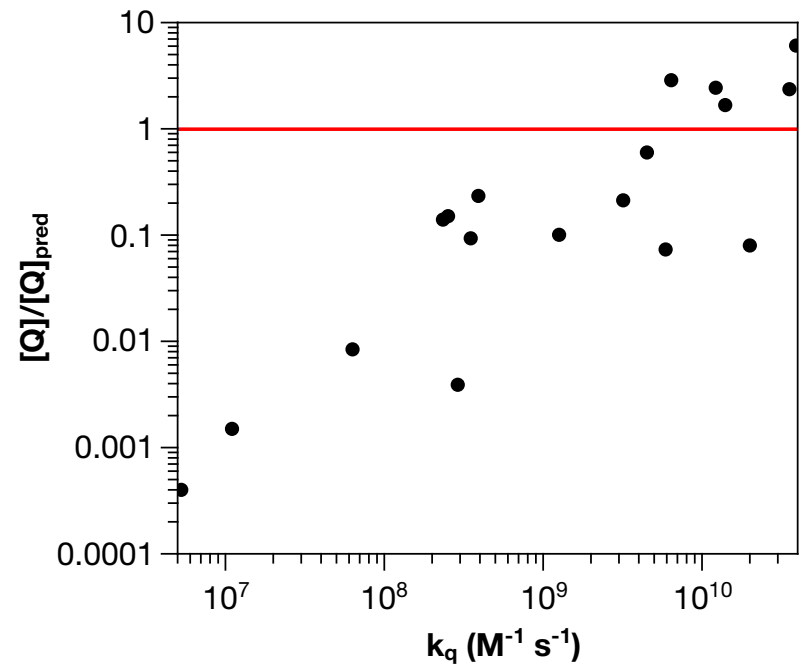

Figure 3. Ratio of experimental quencher concentration to predicted quencher concentration from equation 3 as a function of quenching rate constant, $\mathrm{k}_{\mathrm{q}}$, for eighteen examples of photoredox reactions using acridinium-based photocatalysts. Solid red line indicates a ratio of 1:1 for experimental to predicted quencher concentration. Details for each experimental study are in the supporting information.

In analogy with multi-step synthetic reactions, the overall reaction quantum yield for a photochemical reaction is the product of yield for each individual step. Ensuring a high $\Phi_{\text {quench }}$ offers the best chance of achieving a high quantum yield for the overall reaction and is the step that can be most easily impacted via experimental design. Using kinetic modeling, we have shown that the quencher concentration needed for efficient excited state quenching (90\% or greater) is simply described by equation 3 and relies only on $\tau$ and $\mathrm{k}_{\mathrm{q}}$, two parameters that are readily accessible. Considering the design of photoredox reactions from a quantum yield perspective, equation 3 predicts that optimal photocatalyst lifetimes in the microsecond to tens of microsecond range would be needed in to use quencher concentrations on the order of hundreds of millimolar. 


\section{ASSOCIATED CONTENT}

The following files are available free of charge.

Details of kinetic modeling; comparison of quencher concentrations predicted by equation 3 with those derived from kinetic modeling; details of experimental studies included in Figures 2 and 3 (PDF)

\section{ACKNOWLEDGMENT}

This work was supported by the National Science Foundation (Award Number CHE-2047492). XS thanks the State University of New York Louis Stokes Alliance for Minority Participation (NSF v) for a summer research fellowship.

\section{REFERENCES}

(1) Arias-Rotonda, D. M.; McCusker, J. K. The Photophysics of Photoredox Catalysis: A Roadmap for Catalyst Design. Chem. Sci. Rev. 2016, 45, 5803-5820.

(2) Pitre, S.P.; McTiernan, C.D.; Scaiano, J.C. Understanding the Kinetics and Spectroscopy of Photoredox Catalysis and Transition-Metal-Free Alternatives. Acc. Chem. Res. 2016, 49, 13201330.

(3) Prier, C. K.; Rankic, D. A.; MacMillan, D. W. C. Visible Light Photoredox Catalysis with Transition Metal Complexes: Applications in Organic Synthesis. Chem. Rev. 2013, 113, 53225363. 
(4) Narayanam, J.M.R.; Stephenson, C.R.J. Visible Light Photoredox Catalysis: Applications in Organic Synthesis. Chem. Soc. Rev. 2011, 40, 102-113.

(5) Romero, N. A.; Nicewicz, D. A. Organic Photoredox Catalysis. Chem. Rev. 2016, 116, 10075-10166.

(6) Stevenson, B.G.; Spielvogel, E.H.; Loiaconi, E.A.; Wambua, V. M.; Nakhamiyayev, R.V.; Swierk, J.R. Mechanistic Investigations of an $\alpha$-Aminoarylation Photoredox Reaction. J. Am. Chem. Soc. 2021, 143, 8878-8885.

(7) Spielvogel, E.H.; Stevenson, B.G.; Stringer, M.J.; Swierk, J.R. Insights into the Mechanism of An Allylic Arylation Reaction via Photoredox Coupled Hydrogen Atom Transfer. J. Org. Chem. 2022, 87, 223-230.

(8) W. D. Hinsberg and F. A. Houle, Kinetiscope, available at http://www.hinsberg.net/kinetiscope, accessed May 12, 2019

(9) Liu, M.; Wiegel, A.A.; Wilson, K.R.; Houle, F.A. Aerosol Fragmentation Driven by Coupling of Acid-Base and Free-Radical Chemistry in the Heterogeneous Oxidation of Aqueous Citric Acid by OH Radicals. J. Phys. Chem. A 2017, 121, 5856-5870.

(10) Bolshchikov, B.D.; Tsvetkov, V.B.; Alikhanova, O.L; Serbin, A.V. How to Fight Kinetics in Complex Radical Polymerization Processes: Theoretical Case Study of Poly(divinyl ether-altmaleic anhydride). Macromol. Chem. Phys. 2019, 2019, 1900389.

(11) Bonaldo, F.; Mattivi,F.; Catorci, D.; Arapitsas, P.; Guella, G. H/D Exchange Processes in Flavonoids: Kinetics and Mechanistic Investigations. Molecules 2021, 26, 3544. 
(12) Gehlen, M.H. The Centenary of the Stern-Volmer Equation of Fluorescence Quenching: From the Single Line Plot to the SV Quenching Map. J. Photochem. Photobiol. C: Photochem. Rev. 2020, 42, 100338.

(13) Olmsted, J.; Meyer, T.J. Factors Affecting Cage Escape Yields Following ElectronTransfer Quenching. J. Phys. Chem. 1987, 91, 1649-1655.

(14) Sun, H.; Hoffman, M.Z. Reductive Quenching of the Excited State of Ruthenium(II) Complexes Containing 2,2'-Bipyridine, 2,2'-Bipyrazine, and 2,2'-Bipyramidine. J. Phys. Chem. 1994, 98, 11719-11726.

(15) Aydogan, A.; Bangle, R.E.; Cadranel, A.; Turlington, M.D.; Conroy, D.T.; Cauët, E.; Singleton, M.L.; Meyer, G.J.; Sampaio, R.N.; Elias, B.; Troian-Gautier, L. Accessing Photoredox Transformations with an Iron(III) Photosensitizer and Green Light. J. Am. Chem. Soc. 2021, 143, 15661-15673.

(16) Joshi-Pangu, A.; Lévesque, F.; Roth, H.G.; Oliver, S.F.; Campeau, L.C.; Nicewicz, D.; DiRocco, D.A. Acridinium-Based Photocatalysts: A Sustainable Option in Photoredox Catalysis. J. Org. Chem. 2016, $81,7244-7249$

(17) Ruccolo, S.; Qin, Y.; Schnedermann, C.; Nocera, D. G. General Strategy for Improving the Quantum Efficiency of Photoredox Hydroamidation Catalysis. J. Am. Chem. Soc. 2018, 140, $14926-14937$. 